\title{
THE SCHWARZ TYPE LEMMAS AND THE LANDAU TYPE THEOREM OF MAPPINGS SATISFYING POISSON'S EQUATIONS
}

\author{
SHAOLIN CHEN AND DAVID KALAJ
}

\begin{abstract}
For a given continuous function $g: \Omega \rightarrow \mathbb{C}$, we establish some Schwarz type Lemmas for mappings $f$ in $\Omega$ satisfying the PDE: $\Delta f=g$, where $\Omega$ is a subset of the complex plane $\mathbb{C}$. Then we apply these results to obtain a Landau type theorem, which is a partial answer to the open problem in [13].
\end{abstract}

\section{Preliminaries AND main Results}

Let $\mathbb{C} \cong \mathbb{R}^{2}$ be the complex plane. For $a \in \mathbb{C}$ and $r>0$, we let $\mathbb{D}(a, r)=\{z$ : $|z-a|<r\}$ so that $\mathbb{D}_{r}:=\mathbb{D}(0, r)$ and thus, $\mathbb{D}:=\mathbb{D}_{1}$ denotes the open unit disk in the complex plane $\mathbb{C}$. Let $\mathbb{T}=\partial \mathbb{D}$ be the boundary of $\mathbb{D}$. We denote by $\mathcal{C}^{m}(\Omega)$ the set of all complex-valued $m$-times continuously differentiable functions from $\Omega$ into $\mathbb{C}$, where $\Omega$ is a subset of $\mathbb{C}$ and $m \in \mathbb{N}_{0}:=\mathbb{N} \cup\{0\}$. In particular, let $\mathcal{C}(\Omega):=\mathcal{C}^{0}(\Omega)$, the set of all continuous functions defined in $\Omega$.

For a real $2 \times 2$ matrix $A$, we use the matrix norm $\|A\|=\sup \{|A z|:|z|=1\}$ and the matrix function $\lambda(A)=\inf \{|A z|:|z|=1\}$. For $z=x+i y \in \mathbb{C}$, the formal derivative of the complex-valued functions $f=u+i v$ is given by

$$
D_{f}=\left(\begin{array}{ll}
u_{x} & u_{y} \\
v_{x} & v_{y}
\end{array}\right) \text {, }
$$

so that

$$
\left\|D_{f}\right\|=\left|f_{z}\right|+\left|f_{\bar{z}}\right| \text { and } \lambda\left(D_{f}\right)=|| f_{z}|-| f_{\bar{z}}||
$$

where

$$
f_{z}=\frac{\partial f}{\partial z}=\frac{1}{2}\left(f_{x}-i f_{y}\right) \text { and } f_{\bar{z}}=\frac{\partial f}{\partial \bar{z}}=\frac{1}{2}\left(f_{x}+i f_{y}\right)
$$

We use

$$
J_{f}:=\operatorname{det} D_{f}=\left|f_{z}\right|^{2}-\left|f_{\bar{z}}\right|^{2}
$$

to denote the Jacobian of $f$ and

$$
\Delta f:=\frac{\partial^{2} f}{\partial x^{2}}+\frac{\partial^{2} f}{\partial y^{2}}=4 f_{z \bar{z}}
$$

is the Laplacian of $f$.

File: shaolinkalaj.tex, printed: 23-8-2017, 1.22

2000 Mathematics Subject Classification. Primary: 30H10, 30C62; Secondary: 31A05, 31C05.

Key words and phrases. Schwarz's Lemma, Landau type theorem, Poisson's equation. 
For $z, w \in \mathbb{D}$ with $z \neq w$ and $|z|+|w| \neq 0$, let

$$
G(z, w)=\log \left|\frac{1-z \bar{w}}{z-w}\right| \text { and } P\left(z, e^{i t}\right)=\frac{1-|z|^{2}}{\left|1-z e^{-i t}\right|^{2}}
$$

be the Green function and Poisson kernel, respectively.

Let $\psi: \mathbb{T} \rightarrow \mathbb{C}$ be a bounded integrable function and let $g \in \mathcal{C}(\mathbb{D})$. For $z \in \mathbb{D}$, the solution to the Poisson's equation

$$
\Delta f(z)=g(z)
$$

satisfying the boundary condition $\left.f\right|_{\mathbb{T}}=\psi \in L^{1}(\mathbb{T})$ is given by

$$
f(z)=\mathcal{P}_{\psi}(z)-\mathcal{G}_{g}(z)
$$

where

$$
\mathcal{G}_{g}(z)=\frac{1}{2 \pi} \int_{\mathbb{D}} G(z, w) g(w) d A(w), \mathcal{P}_{\psi}(z)=\frac{1}{2 \pi} \int_{0}^{2 \pi} P\left(z, e^{i t}\right) \psi\left(e^{i t}\right) d t,
$$

and $d A(z)$ denotes the Lebesgue measure in $\mathbb{D}$. It is well known that if $\psi$ and $g$ are continuous in $\mathbb{T}$ and in $\overline{\mathbb{D}}$, respectively, then $f=\mathcal{P}_{\psi}-\mathcal{G}_{g}$ has a continuous extension $\tilde{f}$ to the boundary, and $\tilde{f}=\psi$ in $\mathbb{T}$ (see [18, pp. 118-120] and [2, 19, 20, 22]).

Heinz in his classical paper [17] proved the following result, which is called the Schwraz Lemma of complex-valued harmonic functions: If $f$ is a complex-valued harmonic function from $\mathbb{D}$ into itself satisfying the condition $f(0)=0$, then, for $z \in \mathbb{D}$

$$
|f(z)| \leq \frac{4}{\pi} \arctan |z| .
$$

Later, Pavlović [30, Theorem 3.6.1] removed the assumption $f(0)=0$ and improved (1.3) into the following sharp form

$$
\left|f(z)-\frac{1-|z|^{2}}{1+|z|^{2}} f(0)\right| \leq \frac{4}{\pi} \arctan |z|,
$$

where $f$ is a complex-valued harmonic function from $\mathbb{D}$ into itself.

The first aim of this paper is to extend (1.4) into mappings satisfying the Poisson's equation, which is as follows.

Theorem 1. For a given $g \in \mathcal{C}(\overline{\mathbb{D}})$, if $f \in \mathcal{C}^{2}(\mathbb{D})$ satisfies $\Delta f=g$ and $\left.f\right|_{\mathbb{T}}=\psi \in$ $L^{1}(\mathbb{T})$, then, for $z \in \overline{\mathbb{D}}$,

$$
\left|f(z)-\frac{1}{2 \pi} \frac{1-|z|^{2}}{1+|z|^{2}} \int_{0}^{2 \pi} \psi\left(e^{i t}\right) d t\right| \leq \frac{4\left\|\mathcal{P}_{\psi}\right\|_{\infty}}{\pi} \arctan |z|+\frac{\|g\|_{\infty}}{4}\left(1-|z|^{2}\right),
$$

where

$$
\mathcal{P}_{\psi}(z)=\frac{1}{2 \pi} \int_{0}^{2 \pi} P\left(z, e^{i t}\right) \psi\left(e^{i t}\right) d t,\left\|\mathcal{P}_{\psi}\right\|_{\infty}=\sup _{z \in \mathbb{D}}\left|\mathcal{P}_{\psi}(z)\right| \text { and }\|g\|_{\infty}=\sup _{z \in \mathbb{D}}|g(z)| .
$$

If we take $g(z)=-4 M$ and $f(z)=M\left(1-|z|^{2}\right)$ for $z \in \overline{\mathbb{D}}$, then the inequality (1.5) is sharp in $\mathbb{T} \cup\{0\}$, where $M$ is a positive constant. 
The following result is a classical Schwarz Lemma at the boundary.

Theorem A. (see [15]) Let $f$ be a holomorphic function from $\mathbb{D}$ into itself. If $f$ is holomorphic at $z=1$ with $f(0)=0$ and $f(1)=1$, then $f^{\prime}(1) \geq 1$. Moreover, the inequality is sharp.

Theorem A has attracted much attention and has been generalized in various forms ( see [6, 23, 26, 27] for holomorphic functions, and see [21] for harmonic functions). In the following, applying Theorem 1, we establish a Schwarz Lemma at the boundary for mappings satisfying the Poisson's equation, which is a generalization of Theorem A.

Theorem 2. For a given $g \in \mathcal{C}(\overline{\mathbb{D}})$, let $f \in \mathcal{C}^{2}(\mathbb{D}) \cap \mathcal{C}(\mathbb{T})$ be a function of $\mathbb{D}$ into itself satisfying $\Delta f=g$, where $\|g\|_{\infty}<\frac{8}{3 \pi}$. If $f(0)=0$ and, for some $\zeta \in \mathbb{T}$, $\lim _{r \rightarrow 1^{-}}|f(r \zeta)|=1$, then

$$
\liminf _{r \rightarrow 1^{-}} \frac{|f(\zeta)-f(r \zeta)|}{1-r} \geq \frac{2}{\pi}-\frac{3\|g\|_{\infty}}{4}
$$

where $r \in[0,1)$.

In particular, if $\|g\|_{\infty}=0$, then the estimate of (1.6) is sharp.

In [14], Colonna proved a sharp Schwraz-Pick type Lemma of complex-valued harmonic functions, which is as follows: If $f$ is a complex-valued harmonic function from $\mathbb{D}$ into itself, then, for $z \in \mathbb{D}$,

$$
\left\|D_{f}(z)\right\| \leq \frac{4}{\pi} \frac{1}{1-|z|^{2}}
$$

We extend (1.7) into the following form.

Theorem 3. For a given $g \in \mathcal{C}(\overline{\mathbb{D}})$, if $f \in \mathcal{C}^{2}(\mathbb{D})$ satisfies $\Delta f=g$ and $\left.f\right|_{\mathbb{T}}=\psi \in$ $L^{1}(\mathbb{T})$, then, for $z \in \mathbb{D} \backslash\{0\}$,

$$
\left\|D_{f}(z)\right\| \leq \frac{4\left\|\mathcal{P}_{\psi}\right\|_{\infty}}{\pi} \frac{1}{1-|z|^{2}}+2 \mu(|z|)
$$

where

$$
\frac{\|g\|_{\infty}}{4} \leq \mu(|z|)=\frac{\|g\|_{\infty}\left(1-|z|^{2}\right)}{8|z|^{2}}\left[\frac{1+|z|^{2}}{1-|z|^{2}}-\frac{\left(1-|z|^{2}\right)}{2|z|} \log \frac{1+|z|}{1-|z|}\right] \leq \frac{\|g\|_{\infty}}{3}
$$

and $\mu(|z|)$ is decreasing on $|z| \in(0,1)$. In particular, if $z=0$, then

$$
\left\|D_{f}(0)\right\| \lim _{|z| \rightarrow 0^{+}}\left(\frac{4\left\|\mathcal{P}_{\psi}\right\|_{\infty}}{\pi} \frac{1}{1-|z|^{2}}+2 \mu(|z|)\right)=\frac{4}{\pi}\left\|\mathcal{P}_{\psi}\right\|_{\infty}+\frac{2}{3}\|g\|_{\infty} .
$$

Moreover, if $\|g\|_{\infty}=0$, then the extreme functions

$$
f(z)=\frac{2 M \alpha}{\pi} \arg \left(\frac{1+\phi(z)}{1-\phi(z)}\right)
$$


show that the estimate of (1.8) and (1.9) are sharp, where $|\alpha|=1$ and $M>0$ are constants, and $\phi$ is a conformal automorphism of $\mathbb{D}$.

We remark that if $\|g\|_{\infty}=0$ and $\left\|\mathcal{P}_{\psi}\right\|_{\infty}=1$ in Theorem 3, then (1.8) and (1.9) coincide with (1.7).

Let $\mathcal{A}$ denote the set of all analytic functions $f$ defined in $\mathbb{D}$ satisfying the standard normalization: $f(0)=f^{\prime}(0)-1=0$. In the early 20th century, Landau [24] showed that there is a constant $r>0$, independent of $f \in \mathcal{A}$, such that $f(\mathbb{D})$ contains a disk of radius $r$. Let $L_{f}$ be the supremum of the set of positive numbers $r$ such that $f(\mathbb{D})$ contains a disk of radius $r$, where $f \in \mathcal{A}$. Then we call $\inf _{f \in \mathcal{A}} L_{f}$ the Landau-Bloch constant. One of the long standing open problems in geometric function theory is to determine the precise value of the Landau-Bloch constant. It has attracted much attention, see $[4,25,28,29,32]$ and references therein. The Landau theorem is an important tool in geometric function theory of one complex variable (cf. [5, 33]). Unfortunately, for general class of functions, there is no Landau type theorem (see $[7,32])$. In order to obtain some analogs of the Landau type theorem for more general classes of functions, it is necessary to restrict the class of functions considered (cf. $[1,3,7,8,9,10,11,13,32])$. Let's recall some known results as follows.

Theorem B. ([7, Theorem 2]) Let $f$ be a harmonic mapping in $\mathbb{D}$ such that $f(0)=$ $J_{f}(0)-1=0$ and $|f(z)|<M$ for $z \in \mathbb{D}$, where $M$ is a positive constant. Then $f$ is univalent in $\mathbb{D}_{\rho_{0}}$ with $\rho_{0}=\pi^{3} /\left(64 m M^{2}\right)$, and $f\left(\mathbb{D}_{\rho_{0}}\right)$ contains a univalent disk $\mathbb{D}_{R_{0}}$ with

$$
R_{0}=\frac{\pi}{8 M} \rho_{0}=\frac{\pi^{4}}{512 m M^{3}},
$$

where $m \approx 6.85$ is the minimum of the function $\left(3-r^{2}\right) /\left[r\left(1-r^{2}\right)\right]$ for $r \in(0,1)$.

Theorem C. ([1, Theorem 1]) Let $f(z)=|z|^{2} G(z)+K(z)$ be a biharmonic mapping, that is $\Delta(\Delta f)=0$, in $\mathbb{D}$ such that $f(0)=K(0)=J_{f}(0)-1=0$, where $G$ and $K$ are harmonic satisfying $|G(z)|,|K(z)|<M$ for $z \in \mathbb{D}$, where $M$ is a positive constant. Then there is a constant $\rho_{2} \in(0,1)$ so that $f$ is univalent in $\mathbb{D}_{\rho_{2}}$. In specific $\rho_{2}$ satisfies

$$
\frac{\pi}{4 M}-2 \rho_{2} M-2 M\left[\frac{\rho_{2}^{2}}{\left(1-\rho_{2}\right)^{2}}+\frac{1}{\left(1-\rho_{2}\right)^{2}}-1\right]=0
$$

and $f\left(\mathbb{D}_{\rho_{2}}\right)$ contains a disk $\mathbb{D}_{R_{2}}$, where

$$
R_{2}=\frac{\pi}{4 M} \rho_{2}-2 M \frac{\rho_{2}^{3}+\rho_{2}^{2}}{1-\rho_{2}}
$$

For some $g \in \mathcal{C}(\overline{\mathbb{D}})$, let $\mathcal{F}_{g}(\overline{\mathbb{D}})$ denote the class of all complex-valued functions $f \in \mathcal{C}^{2}(\mathbb{D}) \cap \mathcal{C}(\mathbb{T})$ satisfying $\Delta f=g$ and $f(0)=J_{f}(0)-1=0$. We extend Theorems $\mathrm{B}$ and $\mathrm{C}$ into the following from.

Theorem 4. For a given $g \in \mathcal{C}(\overline{\mathbb{D}})$, let $f \in \mathcal{F}_{g}(\overline{\mathbb{D}})$ satisfying $\|g\|_{\infty} \leq M_{1}$ and $\|f\|_{\infty} \leq M_{2}$, where $M_{1} \geq 0$ and $M_{2}>0$ are constants. Then $f$ is univalent in $\mathbb{D}_{r_{0}}$, 
where $r_{0}$ satisfies the following equation

$$
\frac{1}{\frac{4}{\pi} M_{2}+\frac{2}{3} M_{1}}-\frac{4 M_{2}}{\pi} \frac{r_{0}\left(2-r_{0}\right)}{\left(1-r_{0}\right)^{2}}-2 M_{1}\left[\log 4\left(1+r_{0}\right)-\log r_{0}\right]\left(2+r_{0}\right) r_{0}=0 .
$$

Moreover, $f\left(\mathbb{D}_{r_{0}}\right)$ contains an univalent disk $\mathbb{D}_{R_{0}}$ with

$$
R_{0} \geq \frac{2 M_{2}}{\pi} \frac{r_{0}^{2}\left(2-r_{0}\right)}{\left(1-r_{0}\right)^{2}}
$$

Remark 1.1. Theorem 4 gives an affirmative answer to the open problem of [13] for the u-gradient mapping $f \in \mathcal{C}^{2}(\mathbb{D})$. If $g$ is harmonic, then all $f \in \mathcal{F}_{g}(\overline{\mathbb{D}})$ are biharmonic. Furthermore, if $\|g\|_{\infty}=0$, then all $f \in \mathcal{F}_{g}(\overline{\mathbb{D}})$ are harmonic. Hence, Theorem 4 is also a generalization of series known results, such as [1, Theorem 2], [7, Theorems, 3, 4, 5 and 6], [8, Theorems 2 and 3], and so on.

We want to point out that it is failure of Landau type Theorem for $f \in \mathcal{F}_{g}(\overline{\mathbb{D}})$ without any other additional condition. It means that the condition $f(0)=J_{f}(0)-$ $1=0$ is not sufficient to ensure the function of $f$ to the Poisson equation with Landau type theorem, even when $\|g\|_{\infty}=0$. In particular, Gauthier and Pouryayevali [16] proved that it is also failure of Landau's theorem for quasiconformal mappings $f$ defined in $\mathbb{D}$ satisfying $f(0)=J_{f}(0)-1=0$.

Example 1.10. For $g \equiv 1$ and $z=x+i y \in \mathbb{D}$, let $f_{k}(z)=k x+|z|^{2} / 4+i \frac{y}{k}$, where $k \in\{1,2, \ldots\}$. Then, for all $k \in\{1,2, \ldots\}, f_{k}$ is univalent. For all $k \in\{1,2, \ldots\}$, by simple calculations, we see that $J_{f_{k}}(0)-1=f_{k}(0)=0$, and there is no an absolute constant $\rho_{0}>0$ such that $\mathbb{D}_{\rho_{0}}$ belongs to $f_{k}(\mathbb{D})$.

Example 1.11. For $\|g\|_{\infty}=0$ and $z=x+i y \in \mathbb{D}$, let $f_{k}(z)=k x+i \frac{y}{k}$, where $k \in\{1,2, \ldots\}$. For all $k \in\{1,2, \ldots\}$, it is not difficult to see that $f_{k}$ is univalent and $J_{f_{k}}(0)-1=f_{k}(0)=0$. Moreover, for all $k \in\{1,2, \ldots\}, f_{k}(\mathbb{D})$ contains no disk with radius bigger than $1 / k$. Hence, for all $k \in\{1,2, \ldots\}$, there is no an absolute constant $r_{0}>0$ such that $\mathbb{D}_{r_{0}}$ belongs to $f_{k}(\mathbb{D})$.

Corollary 1. Under the same hypothesis of Theorem 4, there is a $r_{0} \in(0,1)$ such that $f$ is bi-Lipschitz in $\mathbb{D}_{r_{0}}$.

The proofs of Theorems 1, 2, 3, 4 and Corollary 1 will be presented in Section 2.

\section{Proofs of the main Results}

Proof of Theorem 1. For a given $g \in \mathcal{C}(\mathbb{D})$, by (1.1), we have

$$
f(z)=\mathcal{P}_{\psi}(z)-\mathcal{G}_{g}(z), z \in \mathbb{D},
$$

where $\mathcal{P}_{\psi}$ and $\mathcal{G}_{g}$ are defined in (1.2). Since $\mathcal{P}_{\psi}$ is harmonic in $\mathbb{D}$, by (1.4), we see that, for $z \in \mathbb{D}$,

$$
\left|\mathcal{P}_{\psi}(z)-\frac{1-|z|^{2}}{1+|z|^{2}} \mathcal{P}_{\psi}(0)\right| \leq \frac{4\left\|\mathcal{P}_{\psi}\right\|_{\infty}}{\pi} \arctan |z|
$$


On the other hand, for a fixed $z \in \mathbb{D}$, let

$$
\zeta=\frac{z-w}{1-\bar{z} w}
$$

which is equivalent to

$$
w=\frac{z-\zeta}{1-\bar{z} \zeta} .
$$

Then

$$
\begin{aligned}
\left|\mathcal{G}_{g}(z)\right| & =\left|\frac{1}{2 \pi} \int_{\mathbb{D}}\left(\log \frac{1}{|\zeta|}\right) g\left(\frac{z-\zeta}{1-\bar{z} \zeta}\right) \frac{\left(1-|z|^{2}\right)^{2}}{|1-\bar{z} \zeta|^{4}} d A(\zeta)\right| \\
& \leq \frac{\|g\|_{\infty}}{2 \pi}\left|\int_{\mathbb{D}}\left(\log \frac{1}{|\zeta|}\right) \frac{\left(1-|z|^{2}\right)^{2}}{|1-\bar{z} \zeta|^{4}} d A(\zeta)\right| \\
& =\left(1-|z|^{2}\right)^{2}\|g\|_{\infty} \int_{0}^{1}\left[\left(\frac{1}{2 \pi} \int_{0}^{2 \pi} \frac{d t}{\left|1-\bar{z} r e^{i t}\right|^{4}}\right) r \log \frac{1}{r}\right] d r \\
& =\left(1-|z|^{2}\right)^{2}\|g\|_{\infty} \int_{0}^{1}\left[\left(\frac{1}{2 \pi} \int_{0}^{2 \pi} \frac{d t}{\left|\left(1-\bar{z} r e^{i t}\right)^{2}\right|^{2}}\right) r \log \frac{1}{r}\right] d r \\
& =\left(1-|z|^{2}\right)^{2}\|g\|_{\infty} \int_{0}^{1}\left[\left(\frac{1}{2 \pi} \int_{0}^{2 \pi}\left|\sum_{n=0}^{\infty}(n+1)(r \bar{z})^{n} e^{i n t}\right|^{2} d t\right) r \log \frac{1}{r}\right] d r \\
& =\left(1-|z|^{2}\right)^{2}\|g\|_{\infty} \int_{0}^{1}\left(r \log \frac{1}{r}\right) \sum_{n=0}^{\infty}(n+1)^{2}|z|^{2 n} r^{2 n} d r \\
& =\left(1-|z|^{2}\right)^{2}\|g\|_{\infty} \sum_{n=0}^{\infty}(n+1)^{2}|z|^{2 n} \int_{0}^{1} r^{2 n+1}\left(\log \frac{1}{r}\right) d r \\
& =\left.\frac{\left(1-|z|^{2}\right)^{2}\|g\|_{\infty}}{4}\left|z \sum_{n=0}^{\infty}\right| z\right|^{2 n} \\
& =\frac{\|g\|_{\infty}}{4}\left(1-|z|^{2}\right) .
\end{aligned}
$$

Hence, by (2.2) and (2.3), we conclude that

$$
\begin{aligned}
\left|f(z)-\frac{1-|z|^{2}}{1+|z|^{2}} \mathcal{P}_{\psi}(0)\right| & \leq\left|\mathcal{P}_{\psi}(z)-\frac{1-|z|^{2}}{1+|z|^{2}} \mathcal{P}_{\psi}(0)\right|+\left|\mathcal{G}_{g}(z)\right| \\
& \leq \frac{4\left\|\mathcal{P}_{\psi}\right\|_{\infty}}{\pi} \arctan |z|+\frac{\|g\|_{\infty}}{4}\left(1-|z|^{2}\right)
\end{aligned}
$$

Now we prove the sharpness part. For $z \in \overline{\mathbb{D}}$, let

$$
g(z)=-4 M \text { and } f(z)=M\left(1-|z|^{2}\right),
$$

where $M$ is a positive constant. Then 


$$
\begin{aligned}
\left|f(0)-\frac{1}{2 \pi} \int_{0}^{2 \pi} \psi\left(e^{i t}\right) d t\right| & =\left|\mathcal{G}_{g}(0)\right|=\left|\frac{1}{2 \pi} \int_{\mathbb{D}}\left(\log \frac{1}{|w|}\right) g(w) d A(w)\right| \\
& =\frac{2 M}{\pi} \int_{0}^{2 \pi} d t \int_{0}^{1} r \log \frac{1}{r} d r \\
& =M \\
& =\frac{\|g\|_{\infty}}{4}
\end{aligned}
$$

which shows (1.5) is sharp at $z=0$. For $z \in \mathbb{T}$, the optimality of (1.5) is obvious. The proof of this theorem is complete.

Proof of Theorem 2. For a given $g \in \mathcal{C}(\overline{\mathbb{D}})$, by (1.1) with $f$ in place of $\psi$, we have

$$
f(z)=\mathcal{P}_{f}(z)-\mathcal{G}_{g}(z), \quad z \in \mathbb{D}
$$

where $\mathcal{P}_{f}$ and $\mathcal{G}_{g}$ are defined in (1.2). Since $f(0)=0$, we see that

$$
\begin{aligned}
\left|\mathcal{P}_{f}(0)\right| & =\left|\mathcal{G}_{g}(0)\right|=\left|\frac{1}{2 \pi} \int_{\mathbb{D}} \log \frac{1}{|w|} g(w) d A(w)\right| \\
& \leq \frac{\|g\|_{\infty}}{2 \pi} \int_{0}^{2 \pi} d t \int_{0}^{1} r \log \frac{1}{r} d r \\
& =\frac{\|g\|_{\infty}}{4} .
\end{aligned}
$$

By (2.4) and Theorem 1, we have

$$
\begin{aligned}
|f(\zeta)-f(r \zeta)| & =\left|f(\zeta)+\mathcal{P}_{f}(0) \frac{1-|z|^{2}}{1+|z|^{2}}-\mathcal{G}_{g}(0) \frac{1-|z|^{2}}{1+|z|^{2}}-f(r \zeta)\right| \\
& \geq 1-\left|f(r \zeta)-\mathcal{P}_{f}(0) \frac{1-|z|^{2}}{1+|z|^{2}}\right|-\left|\mathcal{G}_{g}(0)\right| \frac{1-|z|^{2}}{1+|z|^{2}} \\
& \geq 1-\frac{4}{\pi} \arctan |z|-\frac{\|g\|_{\infty}}{4}\left(1-|z|^{2}\right)-\left|\mathcal{G}_{g}(0)\right| \frac{1-|z|^{2}}{1+|z|^{2}} \\
& \geq 1-\frac{4}{\pi} \arctan |z|-\frac{\|g\|_{\infty}}{4}\left(1-|z|^{2}\right)-\frac{\|g\|_{\infty}}{4} \frac{\left(1-|z|^{2}\right)}{1+|z|^{2}}
\end{aligned}
$$

which, together with L'Hopital's rule, gives that 


$$
\begin{aligned}
\liminf _{r \rightarrow 1^{-}} \frac{\left|f\left(e^{i \theta}\right)-f\left(r e^{i \theta}\right)\right|}{1-r} & \geq \lim _{r \rightarrow 1^{-}} \frac{1-\frac{4}{\pi} \arctan r-\frac{\|g\|_{\infty}}{4}\left(1-r^{2}\right)-\frac{\|g\|_{\infty}}{4} \frac{\left(1-r^{2}\right)}{1+r^{2}}}{1-r} \\
& =\lim _{r \rightarrow 1^{-}}\left[\frac{4}{\pi} \frac{1}{1+r^{2}}-\|g\|_{\infty} \frac{r}{2}-\|g\|_{\infty} \frac{r}{\left(1+r^{2}\right)^{2}}\right] \\
& =\frac{2}{\pi}-\frac{\|g\|_{\infty}}{2}-\frac{\|g\|_{\infty}}{4} \\
& =\frac{2}{\pi}-\frac{3\|g\|_{\infty}}{4},
\end{aligned}
$$

where $z=r \zeta$ and $\zeta \in \mathbb{T}$.

The sharpness part easily follows from [21, Theorem 2.5]. The proof of this theorem is complete. The proof of this theorem is complete.

Theorem D. ([31] or [19, Proposition 2.4]) Let $X$ be an open subset of $\mathbb{R}$, and $\Omega$ be a measure space. Suppose that a function $F: X \times \Omega \rightarrow \mathbb{R}$ satisfies the following conditions:

(1) $F(x, w)$ is a measurable function of $x$ and $w$ jointly, and is integrable over $\omega$, for almost all $x \in X$ held fixed.

(2) For almost all $w \in \Omega, F(x, w)$ is an absolutely continuous function of $x$. (This guarantees that $\partial F(x, w) / \partial x$ exists almost everywhere.)

(3) $\partial F / \partial x$ is locally integrable; that is, for all compact intervals $[a, b]$ contained in $X$ :

$$
\int_{a}^{b} \int_{\Omega}\left|\frac{\partial}{\partial x} F(x, w)\right| d w d x<\infty .
$$

Then $\int_{\Omega} F(x, w) d w$ is an absolutely continuous function of $x$, and for almost every $x \in X$, its derivative exists and is given by

$$
\frac{d}{d x} \int_{\Omega} F(x, w) d w=\int_{\Omega} \frac{\partial}{\partial x} F(x, w) d w .
$$

Proof of Theorem 3. For a given $g \in \mathcal{C}(\overline{\mathbb{D}})$, by (2.1), we have

$$
f(z)=\mathcal{P}_{\psi}(z)-\mathcal{G}_{g}(z), z \in \mathbb{D},
$$

where $\mathcal{P}_{\psi}$ and $\mathcal{G}_{g}$ are the same as in (2.1). Applying [19, Lemma 2.3] and Theorem $\mathrm{D}$, we have

$$
\begin{aligned}
\frac{\partial}{\partial z} \mathcal{G}_{g}(z) & =\frac{1}{2 \pi} \int_{\mathbb{D}} \frac{\partial}{\partial z} G(z, w) g(w) d A(w) \\
& =\frac{1}{4 \pi} \int_{\mathbb{D}} \frac{\left(1-|w|^{2}\right)}{(z-w)(z \bar{w}-1)} g(w) d A(w) \in \mathcal{C}(\mathbb{D})
\end{aligned}
$$


and

$$
\begin{aligned}
\frac{\partial}{\partial \bar{z}} \mathcal{G}_{g}(z) & =\frac{1}{2 \pi} \int_{\mathbb{D}} \frac{\partial}{\partial \bar{z}} G(z, w) g(w) d A(w) \\
& =\frac{1}{4 \pi} \int_{\mathbb{D}} \frac{\left(1-|w|^{2}\right)}{(\bar{z}-\bar{w})(w \bar{z}-1)} g(w) d A(w) \in \mathcal{C}(\mathbb{D}) .
\end{aligned}
$$

For a fixed $z \in \mathbb{D} \backslash\{0\}$, let

$$
\zeta=\frac{z-w}{1-\bar{z} w}
$$

which implies that

$$
w=\frac{z-\zeta}{1-\bar{z} \zeta}, 1-\bar{z} w=\frac{1-|z|^{2}}{1-\bar{z} \zeta} \text { and } 1-|w|^{2}=\frac{\left(1-|\zeta|^{2}\right)\left(1-|z|^{2}\right)}{|1-\bar{z} \zeta|^{2}}
$$

Then, by (2.5), (2.6) and the change of variables, we have

$$
\begin{aligned}
\left|\frac{\partial}{\partial z} \mathcal{G}_{g}(z)\right| & \leq \frac{1}{4 \pi} \int_{\mathbb{D}} \frac{\left(1-|w|^{2}\right)}{|z-w||z \bar{w}-1|}|g(w)| d A(w) \\
& \leq \frac{\|g\|_{\infty}}{4 \pi} \int_{\mathbb{D}} \frac{\left(1-|w|^{2}\right)}{|z-w| z \bar{w}-1 \mid} d A(w) \\
& =\frac{\|g\|_{\infty}}{4 \pi} \int_{\mathbb{D}} \frac{\left(1-|w|^{2}\right)}{|\zeta||1-\bar{z} w|^{2}} \frac{\left(1-|z|^{2}\right)^{2}}{|1-\bar{z} \zeta|^{4}} d A(\zeta) \\
& =\frac{\|g\|_{\infty}}{4 \pi} \int_{\mathbb{D}} \frac{\left(1-|z|^{2}\right)\left(1-|\zeta|^{2}\right)}{|\zeta||1-\bar{z} \zeta|^{4}} d A(\zeta) \\
& =\frac{\|g\|_{\infty}\left(1-|z|^{2}\right)}{2} \int_{0}^{1}\left[\left(1-r^{2}\right)\left(\frac{1}{2 \pi} \int_{0}^{2 \pi} \frac{d t}{\left|1-\bar{z} r e^{i t}\right|}\right)\right] d r \\
& =\frac{\|g\|_{\infty}\left(1-|z|^{2}\right)}{2} \int_{0}^{1}\left[\left(1-r^{2}\right)\left(\frac{1}{2 \pi} \int_{0}^{2 \pi}\left|\sum_{n=0}^{\infty}(n+1)(r \bar{z})^{n} e^{i n t}\right|^{2} d t\right)\right] d r \\
& =\frac{\|g\|_{\infty}\left(1-|z|^{2}\right)}{2} \int_{0}^{1}\left(1-r^{2}\right)\left[\sum_{n=0}^{\infty}(n+1)^{2}|z|^{2 n} r^{2 n}\right] d r \\
& =\frac{\|g\|_{\infty}\left(1-|z|^{2}\right)}{2} \int_{0}^{1} \frac{\left(1-r^{2}\right)\left(1+|z|^{2} r^{2}\right)}{\left(1-|z|^{2} r^{2}\right)^{3}} d r \\
& =\frac{\|g\|_{\infty}\left(1-|z|^{2}\right)}{2}\left[-\frac{1}{|z|^{2}} I_{1}+\left(\frac{3}{|z|^{2}}-1\right) I_{2}+2\left(1-\frac{1}{|z|^{2}}\right) I_{3}\right], \\
(2.7) &
\end{aligned}
$$

where

$$
I_{1}=\int_{0}^{1} \frac{d r}{1-r^{2}|z|^{2}}=\left.\frac{1}{|z|} \log \frac{1+|z| r}{\sqrt{1-|z|^{2} r^{2}}}\right|_{0} ^{1}=\frac{1}{|z|} \log \frac{1+|z|}{\sqrt{1-|z|^{2}}}
$$




$$
\begin{aligned}
I_{2}=\int_{0}^{1} \frac{d r}{\left(1-r^{2}|z|^{2}\right)^{2}} & =\left.\frac{1}{2|z|}\left(\log \frac{1+|z| r}{\sqrt{1-|z|^{2} r^{2}}}+\frac{|z| r}{1-|z|^{2} r^{2}}\right)\right|_{0} ^{1} \\
& =\frac{1}{2|z|} \log \frac{1+|z|}{\sqrt{1-|z|^{2}}}+\frac{1}{2\left(1-|z|^{2}\right)}
\end{aligned}
$$

and

$$
\begin{aligned}
I_{3}=\int_{0}^{1} \frac{d r}{\left(1-r^{2}|z|^{2}\right)^{3}} & =\left.\frac{1}{4|z|}\left(\frac{|z| r}{\left(1-r^{2}|z|^{2}\right)^{2}}+\frac{3}{2} \frac{|z| r}{1-r^{2}|z|^{2}}+\frac{3}{2} \log \frac{1+|z| r}{\sqrt{1-|z|^{2} r^{2}}}\right)\right|_{0} ^{1} \\
& =\frac{1}{4\left(1-|z|^{2}\right)^{2}}+\frac{3}{8\left(1-|z|^{2}\right)}+\frac{3}{8|z|} \log \frac{1+|z|}{\sqrt{1-|z|^{2}}}
\end{aligned}
$$

By (2.8), (2.9) and (2.10), we get

$$
\begin{aligned}
-\frac{1}{|z|^{2}} I_{1}+\left(\frac{3}{|z|^{2}}-1\right) I_{2}+2\left(1-\frac{1}{|z|^{2}}\right) I_{3}= & \frac{1}{4|z|^{2}}\left[\frac{1+|z|^{2}}{1-|z|^{2}}\right. \\
& \left.-\frac{\left(1-|z|^{2}\right)}{2|z|} \log \frac{1+|z|}{1-|z|}\right],
\end{aligned}
$$

which, together with (2.7), yields that

$$
\left|\frac{\partial}{\partial z} \mathcal{G}_{g}(z)\right| \leq \mu(|z|),
$$

where

$$
\mu(|z|)=\frac{\|g\|_{\infty}\left(1-|z|^{2}\right)}{8|z|^{2}}\left[\frac{1+|z|^{2}}{1-|z|^{2}}-\frac{\left(1-|z|^{2}\right)}{2|z|} \log \frac{1+|z|}{1-|z|}\right] .
$$

By a similar proof process of (2.11), we have

$$
\left|\frac{\partial}{\partial \bar{z}} \mathcal{G}_{g}(z)\right| \leq \mu(|z|)
$$

By direct calculation (or by [19, Lemma 2.3]), we obtain

$$
\begin{gathered}
\lim _{|z| \rightarrow 0^{+}} \frac{\|g\|_{\infty}\left(1-|z|^{2}\right)}{8|z|^{2}}\left[\frac{1+|z|^{2}}{1-|z|^{2}}-\frac{\left(1-|z|^{2}\right)}{2|z|} \log \frac{1+|z|}{1-|z|}\right]=\frac{\|g\|_{\infty}}{3}, \\
\lim _{|z| \rightarrow 1^{-}} \frac{\|g\|_{\infty}\left(1-|z|^{2}\right)}{8|z|^{2}}\left[\frac{1+|z|^{2}}{1-|z|^{2}}-\frac{\left(1-|z|^{2}\right)}{2|z|} \log \frac{1+|z|}{1-|z|}\right]=\frac{\|g\|_{\infty}}{4}
\end{gathered}
$$

and $\mu(|z|)$ is decreasing on $|z| \in(0,1)$. 
On the other hand, since $\mathcal{P}_{\psi}$ is harmonic in $\mathbb{D}$, by [14, Theorem 3] (see also $[11,12])$, we see that, for $z \in \mathbb{D}$,

$$
\left\|D_{\mathcal{P}_{\psi}}(z)\right\| \leq \frac{4\left\|\mathcal{P}_{\psi}\right\|_{\infty}}{\pi} \frac{1}{1-|z|^{2}}
$$

Hence (1.8) follows from (2.11), (2.12) and (2.14). Furthermore, applying (1.8) and (2.13), we get (1.9). The proof of this theorem is complete.

New wi formulate the following well-known result

Lemma 1. The improper integral

$$
\int_{0}^{\frac{\pi}{2}} \log \sin x d x=\int_{0}^{\frac{\pi}{2}} \log \cos x d x=-\frac{\pi}{2} \log 2 .
$$

Lemma 2. For $z \in \mathbb{D} \backslash\{0\}$, the improper integral

$$
\begin{aligned}
\int_{\mathbb{D}} \frac{d A(w)}{|w||z-w|}= & \int_{0}^{2 \pi} \log \left(1-r \cos t+\sqrt{1+r^{2}-2 r \cos t}\right) d t \\
& -2 \pi \log r+2 \pi \log 2 \\
\leq & 2 \pi \log 4(1+r)-2 \pi \log r,
\end{aligned}
$$

where $r=|z|$.

Proof. Let $z=r e^{i \alpha}$ and $w=\rho e^{i \theta}$. Then

$$
\begin{aligned}
\int_{\mathbb{D}} \frac{d A(w)}{|w||z-w|}= & \int_{0}^{1} d \rho \int_{0}^{2 \pi} \frac{d \theta}{\sqrt{r^{2}+\rho^{2}-2 \rho r \cos (\theta-\alpha)}} \\
= & \int_{0}^{1} d \rho \int_{0}^{2 \pi} \frac{d t}{\sqrt{r^{2}+\rho^{2}-2 \rho r \cos t}} \\
= & \int_{0}^{2 \pi} d t \int_{0}^{1} \frac{d \rho}{\sqrt{r^{2}+\rho^{2}-2 \rho r \cos t}} \\
= & \int_{0}^{2 \pi}\left\{\frac { 1 } { 2 r \operatorname { c o s } t } \left[\int_{0}^{1} \frac{2 \rho d \rho}{\sqrt{r^{2}+\rho^{2}-2 \rho r \cos t}}\right.\right. \\
= & \int_{0}^{2 \pi}\left[\frac{1}{r \cos t} \int_{0}^{1} \frac{\rho\left(r^{2}+\rho^{2}-2 \rho r \cos t\right)}{\sqrt{r^{2}+\rho^{2}-2 \rho r \cos t}}\right] d t \\
= & \left.-\frac{1}{r \cos t}\left(\sqrt{1+r^{2}-2 r \cos t}-r\right)\right] d t \\
& \int_{0}^{2 \pi}\left[\frac{1}{r \cos t} \int_{0}^{1} \frac{\rho d \rho}{\sqrt{r^{2}+\rho^{2}-2 \rho r \cos t}}\right. \\
& -\frac{\left.\sqrt{1+r^{2}-2 r \cos t}+\frac{1}{\cos t}\right] d t .}{r \cos t}
\end{aligned}
$$


By calculations, we get

$$
\begin{aligned}
\int_{0}^{1} \frac{\rho d \rho}{\sqrt{r^{2}+\rho^{2}-2 \rho r \cos t}}= & \left.H(\rho)\right|_{0} ^{1} \\
= & \sqrt{1+r^{2}-2 r \cos t} \\
& +r \cos t \log \left(1-r \cos t+\sqrt{1+r^{2}-2 r \cos t}\right) \\
& -r-r \cos t \log r(1-\cos t),
\end{aligned}
$$

where

$$
H(\rho)=\sqrt{\rho^{2}+r^{2}-2 r \rho \cos t}+r \cos t \log \left(\rho-r \cos t+\sqrt{r^{2}+\rho^{2}-2 \rho r \cos t}\right) .
$$

By (2.15), (2.16) and Lemma 1, we see that

$$
\begin{aligned}
\int_{\mathbb{D}} \frac{d A(w)}{|w||z-w|}= & \int_{0}^{2 \pi} \log \left(1-r \cos t+\sqrt{1+r^{2}-2 r \cos t}\right) d t \\
& -\int_{0}^{2 \pi} \log r(1-\cos t) d t \\
= & \int_{0}^{2 \pi} \log \left(1-r \cos t+\sqrt{1+r^{2}-2 r \cos t}\right) d t \\
& -2 \pi \log r-\int_{0}^{2 \pi} \log \left(2 \sin ^{2} \frac{t}{2}\right) d t \\
= & \int_{0}^{2 \pi} \log \left(1-r \cos t+\sqrt{1+r^{2}-2 r \cos t}\right) d t \\
& -2 \pi \log 2 r-8 \int_{0}^{\frac{\pi}{2}} \log (\sin t) d t \\
= & \int_{0}^{2 \pi} \log \left(1-r \cos t+\sqrt{1+r^{2}-2 r \cos t}\right) d t \\
& -2 \pi \log r+2 \pi \log 2 \\
\leq & 2 \pi \log 4(1+r)-2 \pi \log r .
\end{aligned}
$$

The proof of this theorem is complete.

Lemma E. ([10, Lemma 1]) Let $f$ be a harmonic mapping of $\mathbb{D}$ into $\mathbb{C}$ such that $|f(z)| \leq M$ and $f(z)=\sum_{n=0}^{\infty} a_{n} z^{n}+\sum_{n=1}^{\infty} \bar{b}_{n} \bar{z}^{n}$. Then $\left|a_{0}\right| \leq M$ and for all $n \geq 1$,

$$
\left|a_{n}\right|+\left|b_{n}\right| \leq \frac{4 M}{\pi}
$$

Lemma 3. For $x \in(0,1)$, let

$$
\phi(x)=\frac{1}{\frac{4}{\pi} M_{2}+\frac{2}{3} M_{1}}-\frac{4 M_{2}}{\pi} \frac{x(2-x)}{(1-x)^{2}}-2 M_{1}[\log 4(1+x)-\log x](2+x) x,
$$


where $M_{2}>0$ and $M_{1} \geq 0$ are constant. Then $\phi$ is strictly decreasing and there is an unique $x_{0} \in(0,1)$ such that $\phi\left(x_{0}\right)=0$.

Proof. For $x \in(0,1)$, let

$$
f_{1}(x)=\frac{4 M_{2}}{\pi} \frac{x(2-x)}{(1-x)^{2}}
$$

and

$$
f_{2}(x)=2 M_{1}[\log 2(1+x)-\log x+\log 2](2+x) x .
$$

Since, for $x \in(0,1)$,

$$
f_{1}^{\prime}(x)=\frac{8 M_{2}}{\pi} \frac{1}{(1-x)^{3}}>0
$$

and

$$
\begin{aligned}
f_{2}^{\prime}(x) & =2 M_{1}\left[2(x+1) \log \frac{4(1+x)}{x}-\frac{2+x}{1+x}\right] \\
& =2 M_{1}\left\{2(x+1)\left[\log 4+\log \left(1+\frac{1}{x}\right)\right]-\frac{2+x}{1+x}\right\} \\
& \geq 2 M_{1}\left\{2(x+1)\left[1+\frac{1}{1+x}\right]-\frac{2+x}{1+x}\right\} \\
& =2 M_{1} \frac{(2+x)(2 x+1)}{1+x} \geq 0
\end{aligned}
$$

we see that $f_{1}+f_{2}$ is continuous and strictly increasing in $(0,1)$. Then $\phi$ is continuous and strictly decreasing in $(0,1)$, which, together with

$$
\lim _{x \rightarrow 0^{+}} \phi(x)=\frac{1}{\frac{4}{\pi} M_{2}+\frac{2}{3} M_{1}} \text { and } \lim _{x \rightarrow 1^{-}} \phi(x)=-\infty,
$$

implies that there is an unique $x_{0} \in(0,1)$ such that $\phi\left(x_{0}\right)=0$.

Lemma 4. For $x \in(0,1]$, let

$$
\tau_{1}(x)=\frac{2-r_{0} x}{\left(1-r_{0} x\right)^{2}} \text { and } \tau_{2}(x)=x\left[\log 4\left(1+r_{0} x\right)-\log \left(r_{0} x\right)\right],
$$

where $r_{0} \in(0,1)$ is a constant. Then $\tau_{1}$ and $\tau_{2}$ are increasing functions in $(0,1]$.

Proof of Theorem 4. As before, by (2.1) with $f$ in place of $\psi$, we have

$$
f(z)=\mathcal{P}_{f}(z)-\mathcal{G}_{g}(z), z \in \mathbb{D},
$$

where $\mathcal{P}_{f}$ and $\mathcal{G}_{g}$ are defined in (2.1). By [19, Lemma 2.3], Theorem D and Lemma 2 , we have 


$$
\begin{aligned}
(2.18)\left|\frac{\partial \mathcal{G}_{g}(z)}{\partial z}-\frac{\partial \mathcal{G}_{g}(0)}{\partial z}\right|= & \mid \frac{1}{4 \pi} \int_{\mathbb{D}} \frac{\left(1-|w|^{2}\right)}{(z-w)(z \bar{w}-1)} g(w) d A(w) \\
& -\frac{1}{4 \pi} \int_{\mathbb{D}} \frac{\left(1-|w|^{2}\right)}{w} g(w) d A(w) \mid \\
= & \left|\frac{1}{4 \pi} \int_{\mathbb{D}} \frac{z\left(1-|w|^{2}\right)\left(1+|w|^{2}-z \bar{w}\right)}{w(z-w)(z \bar{w}-1)} g(w) d A(w)\right| \\
\leq & \frac{M_{1}|z|}{4 \pi} \int_{\mathbb{D}} \frac{\left.\left(1-|w|^{2}\right)|1+| w\right|^{2}-z \bar{w} \mid}{|w||z-w||1-z \bar{w}|} d A(w) \\
\leq & \frac{|z|(2+|z|) M_{1}}{4 \pi} \int_{\mathbb{D}} \frac{(1+|w|)}{|w||z-w|} d A(w) \\
\leq & \frac{|z|(2+|z|) M_{1}}{2 \pi} \int_{\mathbb{D}} \frac{1}{|w||z-w|} d A(w) \\
\leq & M_{1}[\log 4(1+|z|)-\log |z|]|z|(2+|z|) .
\end{aligned}
$$

By a similar proof process of (2.18), we get

$$
\begin{aligned}
\left|\frac{\partial \mathcal{G}_{g}(z)}{\partial \bar{z}}-\frac{\partial \mathcal{G}_{g}(0)}{\partial \bar{z}}\right|= & \mid \frac{1}{4 \pi} \int_{\mathbb{D}} \frac{\left(1-|w|^{2}\right)}{(\bar{z}-\bar{w})(w \bar{z}-1)} g(w) d A(w) \\
& -\frac{1}{4 \pi} \int_{\mathbb{D}} \frac{\left(1-|w|^{2}\right)}{\bar{w}} g(w) d A(w) \mid \\
\leq & M_{1}[\log 4(1+|z|)-\log |z|]|z|(2+|z|) .
\end{aligned}
$$

On the other hand, $\mathcal{P}_{f}$ can be written by

$$
\mathcal{P}_{f}(z)=\sum_{n=0}^{\infty} a_{n} z^{n}+\sum_{n=1}^{\infty} \bar{b}_{n} \bar{z}^{n}
$$

because $\mathcal{P}_{f}$ is harmonic in $\mathbb{D}$.

Since $\left|\mathcal{P}_{f}(z)\right| \leq M_{2}$ for $z \in \mathbb{D}$, by Lemma E, we have

$$
\left|a_{n}\right|+\left|b_{n}\right| \leq \frac{4 M_{2}}{\pi}
$$

for $n \geq 1$.

By (2.20), we see that 


$$
\begin{aligned}
\left|\frac{\partial \mathcal{P}_{f}(z)}{\partial z}-\frac{\partial \mathcal{P}_{f}(0)}{\partial z}\right|+\left|\frac{\partial \mathcal{P}_{f}(z)}{\partial \bar{z}}-\frac{\partial \mathcal{P}_{f}(0)}{\partial \bar{z}}\right| & =\left|\sum_{n=2}^{\infty} n a_{n} z^{n-1}\right|+\left|\sum_{n=2}^{\infty} n b_{n} \bar{z}^{n-1}\right| \\
& \leq \sum_{n=2}^{\infty} n\left(\left|a_{n}\right|+\left|b_{n}\right|\right)|z|^{n-1} \\
& \leq \frac{4 M_{2}}{\pi} \sum_{n=2}^{\infty} n|z|^{n-1} \\
& =\frac{4 M_{2}}{\pi} \frac{|z|(2-|z|)}{(1-|z|)^{2}} .
\end{aligned}
$$

Applying Theorem 3, we obtain

$$
1=J_{f}(0)=\left\|D_{f}(0)\right\| \lambda\left(D_{f}(0)\right) \leq \lambda\left(D_{f}(0)\right)\left(\frac{4}{\pi} M_{2}+\frac{2}{3} M_{1}\right)
$$

which gives that

$$
\lambda\left(D_{f}(0)\right) \geq \frac{1}{\frac{4}{\pi} M_{2}+\frac{2}{3} M_{1}}
$$

In order to prove the univalence of $f$ in $\mathbb{D}_{r_{0}}$, we choose two distinct points $z_{1}, z_{2} \in$ $\mathbb{D}_{r_{0}}$ and let $\left[z_{1}, z_{2}\right]$ denote the segment from $z_{1}$ to $z_{2}$ with the endpoints $z_{1}$ and $z_{2}$, where $r_{0}$ satisfies the following equation

$$
\frac{1}{\frac{4}{\pi} M_{2}+\frac{2}{3} M_{1}}-\frac{4 M_{2}}{\pi} \frac{r_{0}\left(2-r_{0}\right)}{\left(1-r_{0}\right)^{2}}-2 M_{1}\left[\log 4\left(1+r_{0}\right)-\log r_{0}\right]\left(2+r_{0}\right) r_{0}=0 .
$$

By (2.18), (2.19), (2.21), (2.22), Lemmas 3 and 4, we have 


$$
\begin{aligned}
\left|f\left(z_{2}\right)-f\left(z_{1}\right)\right|= & \left|\int_{\left[z_{1}, z_{2}\right]} f_{z}(z) d z+f_{\bar{z}}(z) d \bar{z}\right| \\
= & \left|\int_{\left[z_{1}, z_{2}\right]} f_{z}(0) d z+f_{\bar{z}}(0) d \bar{z}\right| \\
& -\left|\int_{\left[z_{1}, z_{2}\right]}\left(f_{z}(z)-f_{z}(0)\right) d z+\left(f_{\bar{z}}(z)-f_{\bar{z}}(0)\right) d \bar{z}\right| \\
\geq & \lambda\left(D_{f}(0)\right)\left|z_{2}-z_{1}\right| \\
& -\int_{\left[z_{1}, z_{2}\right]}\left(\left|f_{z}(z)-f_{z}(0)\right|+\left|f_{\bar{z}}(z)-f_{\bar{z}}(0)\right|\right)|d z| \\
\geq & \lambda\left(D_{f}(0)\right)\left|z_{2}-z_{1}\right| \\
& -\int_{\left[z_{1}, z_{2}\right]}\left(\left|\frac{\partial \mathcal{G}_{g}(z)}{\partial z}-\frac{\partial \mathcal{G}_{g}(0)}{\partial z}\right|+\left|\frac{\partial \mathcal{G}_{g}(z)}{\partial \bar{z}}-\frac{\partial \mathcal{G}_{g}(0)}{\partial \bar{z}}\right|\right)|d z| \\
& -\int_{\left[z_{1}, z_{2}\right]}\left(\left|\frac{\partial \mathcal{P}_{f}(z)}{\partial z}-\frac{\partial \mathcal{P}_{f}(0)}{\partial z}\right|+\left|\frac{\partial \mathcal{P}_{f}(z)}{\partial \bar{z}}-\frac{\partial \mathcal{P}_{f}(0)}{\partial \bar{z}}\right|\right)|d z| \\
> & \left|z_{2}-z_{1}\right|\left\{\lambda\left(D_{f}(0)\right)-\frac{4 M_{2}}{\pi} \frac{r_{0}\left(2-r_{0}\right)}{\left(1-r_{0}\right)^{2}}\right. \\
& \left.-2 M_{1}\left[\log 4\left(1+r_{0}\right)-\log r_{0}\right]\left(2+r_{0}\right) r_{0}\right\} \\
\geq & \left|z_{2}-z_{1}\right|\left\{\frac{1}{\frac{4}{\pi} M_{2}+\frac{2}{3} M_{1}}-\frac{4 M_{2}}{\pi} \frac{r_{0}\left(2-r_{0}\right)}{\left(1-r_{0}\right)^{2}}\right. \\
& \left.-2 M_{1}\left[\log 4\left(1+r_{0}\right)-\log r_{0}\right]\left(2+r_{0}\right) r_{0}\right\} \\
= & 0, \\
23) &
\end{aligned}
$$

which yields that $f\left(z_{2}\right) \neq f\left(z_{1}\right)$. The univalence of $f$ follows from the arbitrariness of $z_{1}$ and $z_{2}$.

Now, for all $\zeta=r_{0} e^{i \theta} \in \partial \mathbb{D}_{r_{0}}$, by (2.18), (2.19), (2.21), (2.22), Lemmas 3 and 4 , we obtain 


$$
\begin{aligned}
& |f(\zeta)-f(0)|=\left|\int_{[0, \zeta]} f_{z}(z) d z+f_{\bar{z}}(z) d \bar{z}\right| \\
& =\left|\int_{[0, \zeta]} f_{z}(0) d z+f_{\bar{z}}(0) d \bar{z}\right| \\
& -\left|\int_{[0, \zeta]}\left(f_{z}(z)-f_{z}(0)\right) d z+\left(f_{\bar{z}}(z)-f_{\bar{z}}(0)\right) d \bar{z}\right| \\
& \geq \lambda\left(D_{f}(0)\right) r_{0} \\
& -\int_{[0, \zeta]}\left(\left|f_{z}(z)-f_{z}(0)\right|+\left|f_{\bar{z}}(z)-f_{\bar{z}}(0)\right|\right)|d z| \\
& \geq \lambda\left(D_{f}(0)\right) r_{0} \\
& -\int_{[0, \zeta]}\left(\left|\frac{\partial \mathcal{G}_{g}(z)}{\partial z}-\frac{\partial \mathcal{G}_{g}(0)}{\partial z}\right|+\left|\frac{\partial \mathcal{G}_{g}(z)}{\partial \bar{z}}-\frac{\partial \mathcal{G}_{g}(0)}{\partial \bar{z}}\right|\right)|d z| \\
& -\int_{[0, \zeta]}\left(\left|\frac{\partial \mathcal{P}_{f}(z)}{\partial z}-\frac{\partial \mathcal{P}_{f}(0)}{\partial z}\right|+\left|\frac{\partial \mathcal{P}_{f}(z)}{\partial \bar{z}}-\frac{\partial \mathcal{P}_{f}(0)}{\partial \bar{z}}\right|\right)|d z| \\
& \geq \frac{r_{0}}{\frac{4}{\pi} M_{2}+\frac{2}{3} M_{1}}-\frac{4 M_{2}}{\pi} \int_{[0, \zeta]} \frac{|z|(2-|z|)}{(1-|z|)^{2}}|d z| \\
& -2 M_{1} \int_{[0, \zeta]}[\log 4(1+|z|)-\log |z|]|z|(2+|z|)|d z| \\
& =\frac{r_{0}}{\frac{4}{\pi} M_{2}+\frac{2}{3} M_{1}}-\frac{4 M_{2} r_{0}^{2}}{\pi} \int_{0}^{1} \frac{t\left(2-r_{0} t\right)}{\left(1-r_{0} t\right)^{2}} d t \\
& -2 M_{1} r_{0}^{2} \int_{0}^{1}\left[\log 4\left(1+r_{0} t\right)-\log \left(r_{0} t\right)\right] t\left(2+r_{0} t\right) d t \\
& \geq \frac{r_{0}}{\frac{4}{\pi} M_{2}+\frac{2}{3} M_{1}}-\frac{4 M_{2} r_{0}^{2}}{\pi} \frac{\left(2-r_{0}\right)}{\left(1-r_{0}\right)^{2}} \int_{0}^{1} t d t \\
& -2 M_{1} r_{0}^{2}\left(2+r_{0}\right) \int_{0}^{1}\left[\log 4\left(1+r_{0} t\right)-\log \left(r_{0} t\right)\right] t d t \\
& \geq r_{0}\left\{\frac{1}{\frac{4}{\pi} M_{2}+\frac{2}{3} M_{1}}-\frac{2 M_{2}}{\pi} \frac{r_{0}\left(2-r_{0}\right)}{\left(1-r_{0}\right)^{2}}\right. \\
& \left.-2 M_{1} r_{0}\left(2+r_{0}\right)\left[\log 4\left(1+r_{0}\right)-\log r_{0}\right]\right\} \\
& =\frac{2 M_{2}}{\pi} \frac{r_{0}^{2}\left(2-r_{0}\right)}{\left(1-r_{0}\right)^{2}} \text {. }
\end{aligned}
$$

Hence $f\left(\mathbb{D}_{r_{0}}\right)$ contains an univalent disk $\mathbb{D}_{R_{0}}$ with

$$
R_{0} \geq \frac{2 M_{2}}{\pi} \frac{r_{0}^{2}\left(2-r_{0}\right)}{\left(1-r_{0}\right)^{2}}
$$

The proof of this theorem is complete. 
Proof of Corollary 1. For $z_{1}, z_{2} \in \mathbb{D}_{r_{0}}$, by (2.23), we see that there is a positive constant $L_{1}$ such that

$$
L_{1}\left|z_{1}-z_{2}\right| \leq\left|f\left(z_{1}\right)-f\left(z_{2}\right)\right|
$$

where $r_{0}$ satisfies the following equation

$$
\frac{1}{\frac{4}{\pi} M_{2}+\frac{2}{3} M_{1}}-\frac{4 M_{2}}{\pi} \frac{r_{0}\left(2-r_{0}\right)}{\left(1-r_{0}\right)^{2}}-2 M_{1}\left[\log 4\left(1+r_{0}\right)-\log r_{0}\right]\left(2+r_{0}\right) r_{0}=0 .
$$

On the other hand, for $z_{1}, z_{2} \in \mathbb{D}_{r_{0}}$, we use Theorem 3 to get

$$
\begin{aligned}
\left|f\left(z_{2}\right)-f\left(z_{1}\right)\right| & =\left|\int_{\left[z_{1}, z_{2}\right]} d f(z)\right| \\
& \leq \int_{\left[z_{1}, z_{2}\right]}|| D_{f}(z) \||d z| \\
& \leq \int_{\left[z_{1}, z_{2}\right]}\left(\frac{4 M_{2}}{\pi} \frac{1}{1-r_{0}^{2}}+\frac{2}{3} M_{1}\right)|d z| \\
& =\left(\frac{4 M_{2}}{\pi} \frac{1}{1-r_{0}^{2}}+\frac{2}{3} M_{1}\right)\left|z_{1}-z_{2}\right|,
\end{aligned}
$$

where $\left[z_{1}, z_{2}\right]$ is the segment from $z_{1}$ to $z_{2}$ with the endpoints $z_{1}$ and $z_{2}$. Therefore, $f$ is bi-Lipschitz in $\mathbb{D}_{r_{0}}$.

\section{REFERENCES}

1. Z. Abdulhadi and Y. Abu Muhanna, Landau's theorem for biharmonic mappings, J. Math. Anal. Appl. 338 (2008), 705-709.

2. K. Astala, T. Iwaniec and G. Martin, Elliptic partial differential equations and quasiconformal mappings in the plane, in: Princeton Mathematical Series, vol. 48, Princeton University Press, Princeton, NJ, 2009, p. xviii +677.

3. M. Bonk, A. Eremenko, Covering properties of meromorphic functions, negative curvature and spherical geometry, Ann. Math., 152(2000), 551-592.

4. M. Bonk, On Bloch's constant, Proc. Amer. Math. Soc., 378(1990), 889-894.

5. R. Brody, Compact manifolds and hyperbolicity, Trans. Amer. Math. Soc., 235(1978), 213219.

6. D. M. Burns And S. G. Krantz, Rigidity of holomorphic mappings and a new Schwarz lemma at the boundary, J. Amer. Math. Soc., 7 (1994), 661-676.

7. H. Chen, P.M. Gauthier, and W. Hengartner, Bloch constants for planar harmonic mappings, Proc. Amer. Math. Soc. 128(2000), 3231-3240.

8. H. Chen And P. M. Gauthier, The landau theorem and Bloch theorem for planar harmonic and pluriharmonic mappings, Proc. Amer. Math. Soc., 139(2011), 583-595.

9. Sh. Chen, S. Ponnusamy and X. Wang, On planar harmonic Lipschitz and planar harmonic Hardy classes, Ann. Acad. Sci. Fenn. Math., 36 (2011), 567-576.

10. Sh. Chen, S. Ponnusamy and X. Wang, Bloch constant and Landau's theorems for planar p-harmonic mappings, J. Math. Anal. Appl., 373(2011), 102-110.

11. Sh. Chen and M. Vuorinen, Some properties of a class of elliptic partial differential operators, J. Math. Anal. Appl., 431 (2015), 1124-1137.

12. Sh. Chen, S. Ponnusamy, A. Rasila and X. Wang, Linear connectivity, Schwarz-Pick lemma and univalency criteria for planar harmonic mappings, Acta Math. Sinica (English Series), 32 (2016), 297-308.

13. Sh. Chen And S. Ponnusamy, Landau's theorem for solutions of the $\bar{\partial}$-equation in Dirichlettype spaces, Bull. Aust. Math. Soc., doi: 10.1017/S0004972717000727, 2017. 
14. F. Colonna, The Bloch constant of bounded harmonic mappings, Indiana Univ. Math. J., 38 (1989), 829-840.

15. J. Garnett, Bounded analytic functions, Academic Press, New York, 1981.

16. P. M. Gauthier and M. R. Pouryayevali, Failure of Landau's theorem for quasiconformal mappings of the disc, Contemporay Math., 355 (2004), 265-268.

17. E. Heinz, On one-to-one harmonic mappings, Pacific J. Math., 9 (1959), 101-105.

18. L. Hörmander, Notions of convexity, Progress in Mathematics, Vol. 127, Birkhäuser Boston Inc, Boston 1994.

19. D. Kalaj And M. Pavlović, On quasiconformal self-mappings of the unit disk satisfying Poisson's equation, Trans. Amer. Math. Soc., 363 (2011), 4043-4061.

20. D. Kalaj, Cauchy transform and Poisson's equation, Adv. Math., 231 (2012), 213-242.

21. D. Kalaj, Heinz-Schwarz inequalities for harmonic mappings in the unit ball, Ann. Acad. Sci. Fenn. Math., 41 (2016), 457-464.

22. D. KalaJ, On some integral operators related to the Poisson equation, Integr. Equ. Oper. Theory, 72 (2012), 563-575.

23. S. G. Krantz, The Schwarz lemma at the boundary, Complex Var. Elliptic Equa., 56 (2011), 455-468.

24. E. Landau, Über die Bloch'sche konstante und zwei verwandte weltkonstanten, Math. Z., 30(1929), 608-634.

25. X. Y. Liu and C. D. Minda, Distortion theorems for Bloch functions, Trans. Amer. Math. Soc. 333(1992), 325-338.

26. T. S. Liu, J. F. WAng And X. M. TAng, Schwarz lemma at the boundary of the unit ball in $\mathbb{C}^{n}$ and its applications, J. Geom. Anal., 25 (2015), 1890-1914.

27. T. S. Liu And X. M. TANG, Schwarz lemma at the boundary of strongly pseudoconvex domain in $\mathbb{C}^{n}$, Math. Ann., 366 (2016), 655-666.

28. D. MindA, Bloch constants, J. Analyse Math. 41(1982), 54-84.

29. D. Minda, Marden constants for Bloch and normal functions, J. Analyse Math. 42(1982/83), $117-127$.

30. M. PAVlović, Introduction to function spaces on the disk, Matematički institut SANU, Belgrade, 2004.

31. E. Talvila, Necessary and sufficient conditions for differentiating under the integral sign, Amer. Math. Monthly 108 (2001), 544-548.

32. H. Wu, Normal families of holomorphic mappings, Acta Math. 119(1967), 193-233.

33. L. Zalcman, Normal families: New perspectives, Bull. Amer. Math. Soc., 35(1998), 215-230.

Sh. Chen, College of Mathematics and Statistics, Hengyang Normal University, Hengyang, Hunan 421008, People's Republic of China.

E-mail address: mathechen@126.com

D. Kalaj, Faculty of Natural Sciences and Mathematics, University of Montenegro, Cetinjski put B. B. 81000 Podgorica, Montenegro.

E-mail address: davidk@t-com.me 